\title{
Study on the soft-start process of PSM high voltage power supply for ECRH
}

\author{
Jian Zhang ${ }^{1}$, Rui Guan ${ }^{1,2}$ Fei Guo ${ }^{1,}$ Yu Zhou ${ }^{1,2}$, Haozhang Sun ${ }^{1}$, Junjiang Wang ${ }^{1,2}$, Yiyun Huang ${ }^{1,2}$ \\ ${ }^{1}$ Hefei Institutes of Physical Science, Chinese Academy of Sciences, Hefei 230031, China \\ ${ }^{2}$ University of Science and Technology of China, Hefei 230026, China
}

\begin{abstract}
The soft-start process of high voltage power supply (HVPS) based on pulse step modulation (PSM) for ECRH on EAST is introduced, which is the first procedure of system operation. The response process is detailed by proposing DC equivalent circuit model, process analysis and performance comparison is given under the conditions of different soft-start resistor parameters, and the theoretical analysis is proved by the simulation package ANSYS Simplorer simulations. The soft-start resistor is designed for the HVPS of $140 \mathrm{GHz}$ ECRH system for a smooth charging without overshoot of the capacitor ${ }^{[6]}$, and the final experimental results show that it is in agreement with the theoretical analysis and is stable and reliable to the power devices.
\end{abstract}

Keywords. EAST, ECRH, pulse step modulation (PSM), soft-start

\section{Introduction}

The HVPS based on PSM ${ }^{[1]}$ technology has the advantages of fast opening and closing, high reliability and modularization etc., which has been widely used in Tokamak auxiliary heating system. The structure of $140 \mathrm{GHz}$ ECRH HVPS system ${ }^{[2,3]}$ is shown in Fig.1, which is composed of $10 \mathrm{kV}$ breaker cabinets, $10 \mathrm{kV}$ contactor cabinets, multi-winding transformers and rectifying modules.

There are two steps to establish output DC voltage of power supply.

1) The first step is that the filter capacitor of rectifier module is charged by closing breaker and contactor.

2) The second step is that the output DC voltage is built by closing IGBT.
When the HVPS is powered up, to limit the inrush current of transformers and the charging current of capacitor, the series resistor needs to be connected in the $10 \mathrm{kVAC}$ side or in the module side. There are almost one hundred of rectifier modules in the HVPS system, so the series resistor ${ }^{[4,5,6]}$ in each module will significantly increase the manufacturing difficulty, volume and cost of modules, therefore the series resistor in $10 \mathrm{kVAC}$ side is more economical and feasible ${ }^{[7]}$. The soft-start includes two processes: insert and removal of the resistor. In the following, the soft-start process will be analyzed theoretically and be verified by ANSYS Simplorer simulation and experimental results.

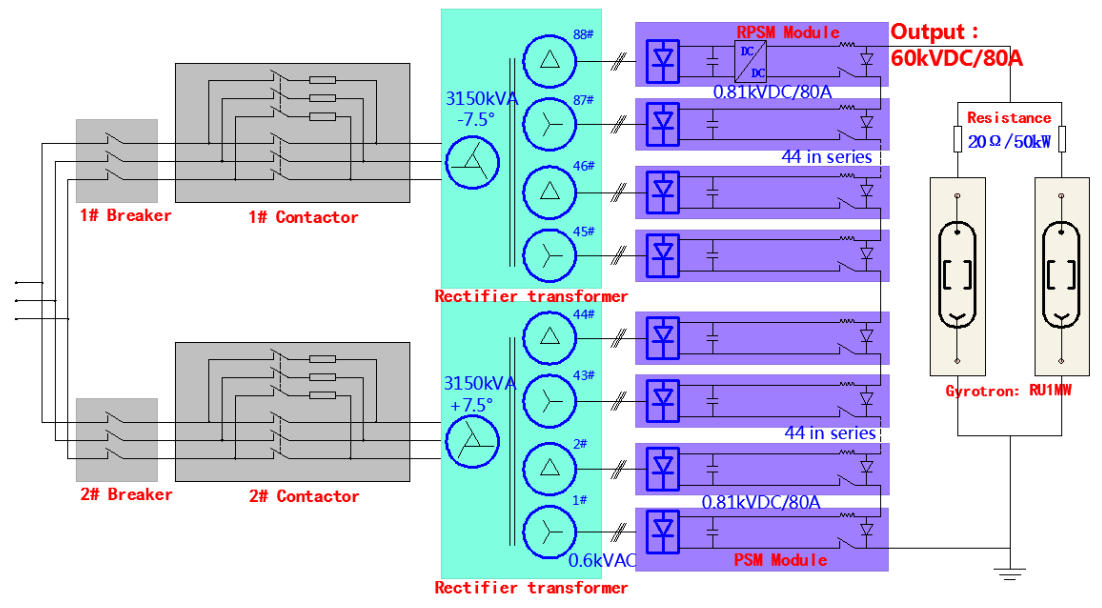

Fig. 1. The topology of HVPS for $140 \mathrm{GHz}$ ECRH system on EAST.

\footnotetext{
* Corresponding author: yyhuang@ipp.ac.cn
} 


\section{DC equivalent model of rectifier module}

The equivalent circuit with the series resistor which is located in the 10kVAC side is shown in Fig.2.

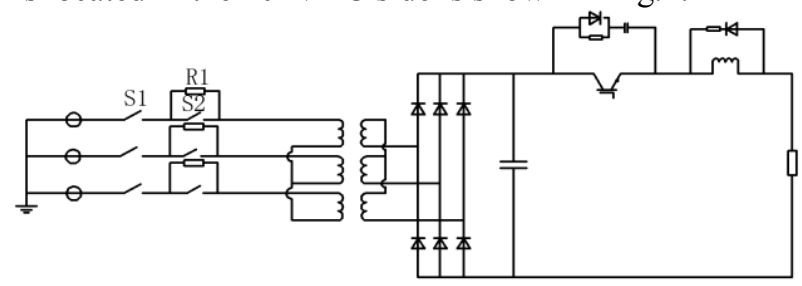

Fig. 2. The equivalent model of rectifying module.

The AC source charges the capacitor through the soft-start resistor, transformer, and three-phase uncontrolled rectifier, and then the DC output of the rectifying module is controlled by the IGBT switch. The process of charging the filter capacitor is called the soft start process. The parameters of each branch are equal, mutual influence can be ignored, and the current and voltage on each branch are approximately equal, so the model in Fig. 2 can be simplified as DC equivalent circuit which is shown in Fig.3.

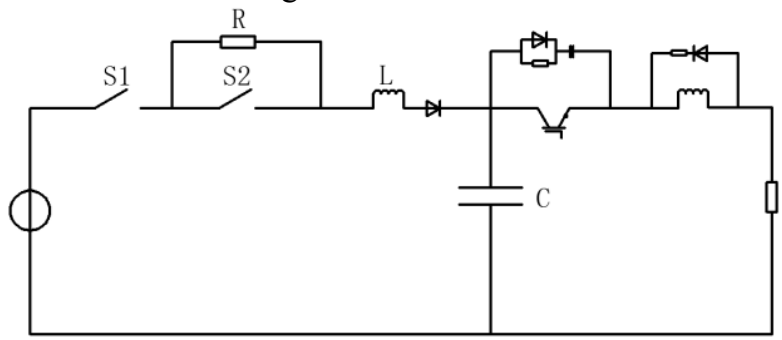

Fig. 3. The DC equivalent circuit of rectifying module.

The basic parameters of multi-winding transformer are shown in Table 1.

Table 1. The basic parameters of multi-winding transformer.

\begin{tabular}{|c|c|c|}
\hline & Primary side & Secondary side \\
\hline Rated power $\mathrm{S}_{\mathrm{N}}$ & \multicolumn{2}{|c|}{$3150 \mathrm{kVA}$} \\
\hline Rated voltage $\mathrm{U}_{\mathrm{NL}}$ & $10 \mathrm{kV}$ & $600 \mathrm{~V}$ \\
\hline Number of Winding & 1 & 44 \\
\hline Short-circuit impedance $\mathrm{U}_{\mathrm{K}}$ & \multicolumn{2}{|c|}{$4 \%$} \\
\hline Field current $\mathrm{I}_{\mathrm{f}}$ & \multicolumn{2}{|c|}{$0.73 \mathrm{~A}$} \\
\hline
\end{tabular}

When the leakage inductance is converted to DC side, the leakage inductance can be calculated by the conventional method of transformer. The leakage inductance is expressed as follow equation:

$$
L^{\prime}=\frac{\left[\frac{\left(\frac{U_{2 N L}}{\sqrt{3}}\right)^{2}}{\frac{S_{N}}{3 N}}\right]^{2}}{2 \pi f}
$$

$U_{2 N L}$ : line voltage of secondary side

$\mathrm{N}$ : number of secondary winding

\footnotetext{
* Corresponding author: yyhuang@ipp.ac.cn
}

Because the current always flows over two phases, when the filter capacitor is charged, the DC side resistor is two times of it in the $\mathrm{AC}$ side.

Equivalent inductance:

$$
\mathrm{L}=2 \mathrm{~L}^{\prime}
$$

Equivalent resistor:

$$
R=\frac{2 N R_{\text {soft }}}{K^{2}}
$$

$\mathrm{R}_{\text {soff: }}$ soft-start resistor

\section{The analysis of soft-start process}

The soft-start process consists of two steps:

1) The first step is that the soft-start resistor is connected into the circuit, the filter capacitor is charged to the first steady state value through the soft-start resistor.

2) The second step is that the soft-start resistor is removed, so the filter capacitor is charged to the second steady state value.

\subsection{The charging process with the soft-start resistor}

The charging equivalent circuit with the soft-start resistor is shown in Fig.4.

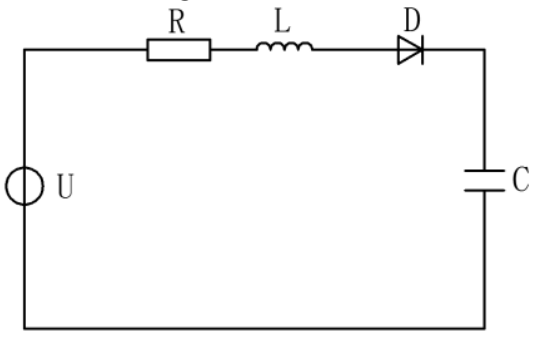

Fig. 4. The charging equivalent circuit with the soft-start resistor.

The transfer function of the filter capacitor voltage and step excitation is established as follow equation.

$$
\frac{U_{C}(s)}{U_{i}(s)}=\frac{1 / L C}{s^{2}+s(R / L)+(1 / L C)}
$$

And the characteristic equation of transfer

function is $s^{2}+s(R / L)+(1 / L C)=0$, so

$$
\Delta=(R / L)^{2}-4 \frac{1}{L C}
$$

When $\Delta<0$, the equivalent resistor satisfies $\mathrm{R}<$ $2 \sqrt{\frac{\mathrm{L}}{\mathrm{C}}}$, the circuit is underdamped. It will cause overshoot of the output voltage. In order to avoid overshoot, $\Delta$ should be more than zero, the equivalent resistor should satisfy $R>2 \sqrt{\frac{L}{C}}$, so that the circuit is overdamped. Take note of (3), the soft-start resistor can be defined by:

$$
\mathrm{R}_{\text {soft }}>\frac{\mathrm{K}^{2}}{\mathrm{~N}} \sqrt{\frac{\mathrm{L}}{\mathrm{C}}}
$$


If the resistor is large enough to satisfyR $\gg 2 \sqrt{\frac{L}{C}}$, the RLC circuit can be equivalent to a RC circuit,

$$
\text { so, } \quad\left\{\begin{array}{c}
\mathrm{U}_{\mathrm{C}}(\mathrm{t})=\mathrm{U}\left(1-\mathrm{e}^{-\frac{1}{\mathrm{RC}} \mathrm{t}}\right) \\
\mathrm{i}_{\mathrm{C}}=\frac{\mathrm{U}}{\mathrm{R}} \mathrm{e}^{-\frac{1}{\mathrm{RC}} \mathrm{t}}
\end{array}\right.
$$

$\mathrm{U}$ : Input voltage of DC equivalent circuit

The peak current of charging is express as follow equation:

$$
i_{\max 1}=\frac{U}{\frac{2 N R_{s o f t}}{K^{2}}}
$$

In order to ensure that the rectifying bridge and capacitor can withstand maximum current, the charging current must be less than $300 \mathrm{~A}$, therefore the soft-start resistor should satisfy:

$$
R_{\text {soft }}>\frac{K^{2} \mathrm{U}}{2 N \times 300}
$$

According to (5), when $\mathrm{N}=1, \frac{K^{2}}{\mathrm{~N}} \sqrt{\frac{L}{C}}$ get the maximum $K^{2} \sqrt{\frac{L}{C}}$, so the soft-start resistor satisfy:

$$
\mathrm{R}_{\text {soft }}>K^{2} \sqrt{\frac{L}{C}}
$$

The circuit is simulated by selecting different softstart resistor, the results are shown in Figure 5. According to the design of the module, the simulation parameters are set as follows:

1) Input voltage of $D C$ equivalent circuit $U: 1000 \mathrm{~V}$. $2 \mathrm{mH}$.

2) Equivalent inductance of DC equivalent circuit $\mathrm{L}$ :

3) Filter capacitor C: $10 \mathrm{mF}$.

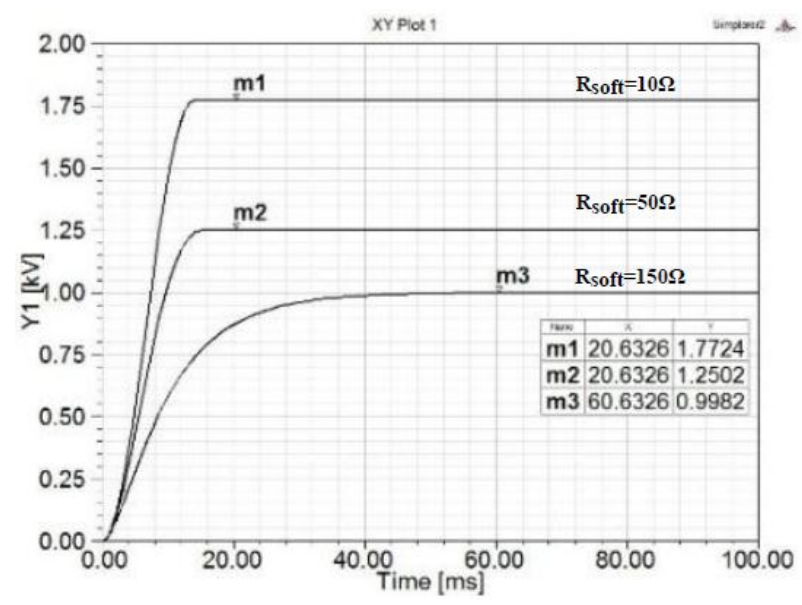

Fig. 5. The capacitor voltage of different soft-start resistor.

Table 2. The Influence of soft-start resistor on charge voltage.

\begin{tabular}{|c|c|c|}
\hline $\begin{array}{c}\mathbf{R}_{\text {soft }} \\
(\boldsymbol{\Omega})\end{array}$ & $\begin{array}{c}\text { Steady time } \\
\mathbf{T}(\mathbf{m s})\end{array}$ & $\begin{array}{c}\text { Steady voltage } \\
\mathbf{U}_{\mathbf{c}}(\mathbf{V})\end{array}$ \\
\hline 10 & 14 & 1772 \\
\hline 50 & 16 & 1250 \\
\hline 150 & 60 & 998 \\
\hline
\end{tabular}

According to Fig.5 and Table 2, when the soft-start resistor is less than $K^{2} \sqrt{\frac{L}{C}}$ which is equal to $124 \Omega$ according to the simulation parameters, the circuit is underdamped, the steady voltage of filter capacitor is larger than $1000 \mathrm{~V}$, and furthermore, the overshoot voltage is smaller with the increase of the soft start resistor. When the soft-start resistor is larger than $K^{2} \sqrt{\frac{L}{C}}$ which is equal to $124 \Omega$, the circuit is over damped, the steady voltage of filter capacitor is about $1000 \mathrm{~V}$, and the capacitor is charged without overshoot.

\subsection{The charging process of removing the soft- start resistor}

When the filter capacitor is charged to the first steady state value, the voltage is less than the rated value because of soft-start resistor voltage drop, so the softstart resistor must be removed to get the rated value and reduce the power loss. The soft-start resistor is removed by closing S2 in Fig.3, then the capacitor will be charged again to the rated value. The equivalent circuit is shown in Fig.6.

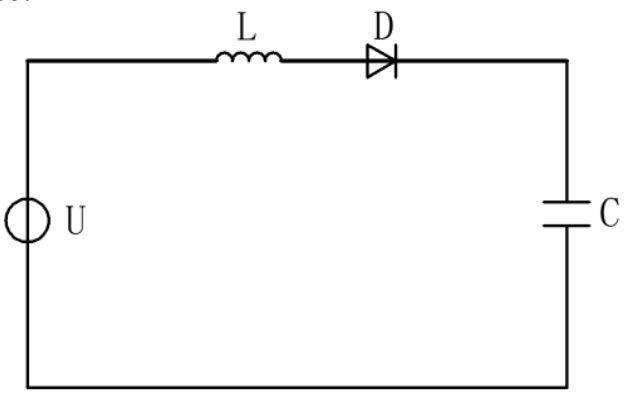

Fig. 6. The removing process of soft-start resistor.

The voltage drop of soft-start resistor which is produced by transformer magnetizing current is $\Delta \mathrm{U}=$ $I_{f} R_{\text {soft }}$, the excitation can be equivalent to:

$$
u_{i}=\frac{\sqrt{2} \sqrt{3} I_{f} R_{\text {soft }}}{K}=\frac{\sqrt{6} I_{f} R_{\text {soft }}}{K}
$$

The time domain expression is established as follow equation.

$$
L C \frac{d^{2} u_{C}}{d t^{2}}+u_{C}=u_{i}
$$

According to the initial condition of filter capacitor: $u_{C}=U-\frac{\sqrt{6} I_{f} R_{s o f t}}{K}, \mathrm{C} \frac{d u_{C}}{d t}=0$, the capacitance voltage and current are expressed as follow:

$$
\left\{\begin{array}{l}
\mathrm{u}_{\mathrm{C}}=\mathrm{U}-\frac{\sqrt{6} \mathrm{I}_{\mathrm{f}} \mathrm{R}_{\text {soft }}}{\mathrm{K}} \cos \frac{\mathrm{t}}{\sqrt{\mathrm{LC}}} \\
\mathrm{i}_{\mathrm{C}}=\frac{\sqrt{6} \mathrm{I}_{\mathrm{f}} \mathrm{R}_{\text {soft }}}{\mathrm{K}} \sqrt{\frac{\mathrm{C}}{\mathrm{L}}} \sin \frac{\mathrm{t}}{\sqrt{\mathrm{LC}}}
\end{array}\right.
$$

According to (10), the voltage increment of filter capacitor is:

$$
\Delta u_{C}=\frac{2 \sqrt{6} I_{f} R_{\text {soft }}}{K}
$$

The peak current is expressed as follow equation:

$$
i_{\max 2}=\frac{\sqrt{6} I_{f} R_{\text {soft }}}{K} \sqrt{\frac{C}{L}}
$$

In order to ensure that the charging current is less than $300 \mathrm{~A}$, the soft-start resistor should satisfy:

$$
R_{\text {soft }}<\frac{300 K}{\sqrt{6} I_{f}} \sqrt{\frac{L}{C}}
$$


The circuit is simulated by selecting different softstart resistor, the selection of resistor need to guarantee that the circuit is over damped. According to the simulation parameters, the soft-start resistor must be $\mathrm{R}_{\text {soft }}>K^{2} \sqrt{\frac{L}{C}}$ and the simulation parameters are set as follows:

1) Input voltage of DC equivalent circuit U: $1000 \mathrm{~V}$. $2 \mathrm{mH}$.

2) Equivalent inductance of DC equivalent circuit L:

3) Filter capacitor $\mathrm{C}: 10 \mathrm{mF}$.

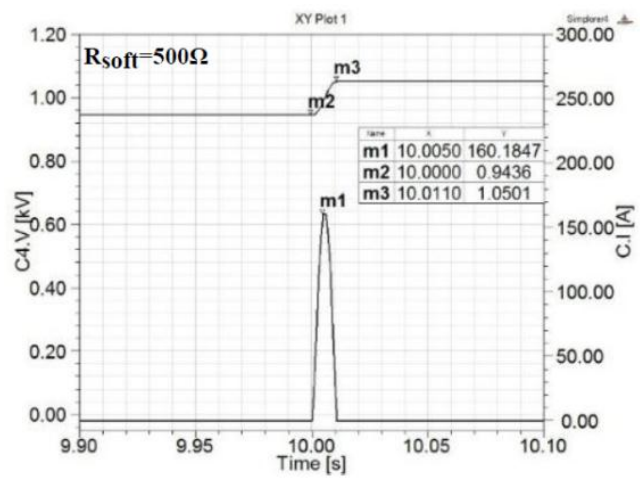

(a) $R_{\text {soft }}=500 \Omega$

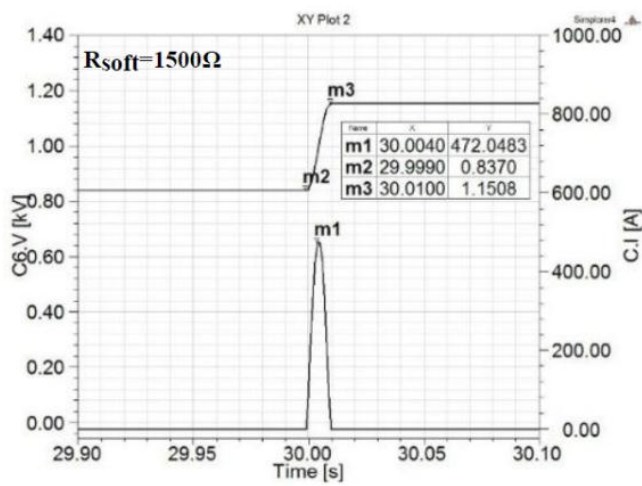

(b) $R_{\text {soft }}=1500 \Omega$

Fig. 7.The capacitor voltage of removing different softstart resistor.

Table 3. The Influence of soft-start resistor on charge voltage and current.

\begin{tabular}{|c|c|c|c|c|}
\hline $\mathbf{R}_{\text {soft }}(\mathbf{\Omega})$ & $\mathbf{I}_{\max \mathbf{2}}(\mathbf{A})$ & $\mathbf{U}_{\mathbf{1}}(\mathbf{V})$ & $\mathbf{U}_{\mathbf{2}}(\mathbf{V})$ & $\boldsymbol{\Delta} \mathbf{U}$ \\
\hline 500 & 160 & 943 & 1050 & 107 \\
\hline 1500 & 472 & 837 & 1150 & 313 \\
\hline
\end{tabular}

$\mathrm{I}_{\max 2}$ : Maximum charging current of removing the softstart resistor.

$\mathrm{U}_{1}$ : The initial voltage of the filter capacitor before removing the soft-start resistor.

$\mathrm{U}_{2}$ : The steady voltage of the filter capacitor after removing the soft-start resistor.

$\Delta \mathrm{U}$ : The voltage change of the filter capacitor by removing the soft-start resistor.
According to (13), when the soft-start resistor is less than $\frac{300 K}{\sqrt{6} I_{f}} \sqrt{\frac{L}{C}}$, which is equal to $968 \Omega$, the rectifying bridge and capacitor can withstand maximum current 300A. Take note of Table 3, when $\mathrm{R}_{\text {soft }}=500<968 \Omega$, $\mathrm{I}_{\max 2}=160<300 \mathrm{~A}$, and when $\mathrm{R}=1500>968 \Omega, \mathrm{I}_{\max 2}=472$ $>300 \mathrm{~A}$. According to (12) and Table $3, \mathrm{I}_{\max 2}$ will enlarge with the increase of the soft-start resistor, and according to (11) and Table 3, $\triangle \mathrm{U}$ will enlarge with the increase of the soft-start resistor. So in order to reduce voltage fluctuation of filter capacitor, the soft-start resistor should be reduced as far as possible.

According to the analysis, the design of the softstart resistor must meet the following conditions.

(1)In order to avoid overshoot, the soft-start resistor must satisfy:

$$
\mathrm{R}_{\text {soft }}>K^{2} \sqrt{\frac{L}{C}} .
$$

(2)In order to ensure that the rectifying bridge and capacitor can withstand the maximum charging current, the soft-start resistor must satisfy:

$$
\frac{K^{2} U_{i}}{2 N \times 300}<R_{\text {soft }}<\frac{300 K}{\sqrt{6} I_{f}} \sqrt{\frac{L}{C}} .
$$

\section{The resistor design of HVPS for ECRH system}

According to the multi-windings transformer parameters in the HVPS for $140 \mathrm{GHz}$ ECRH system, the leakage inductance of transformer can be calculated as follow:

$$
\mathrm{L}_{1}=\frac{\left[\frac{\left(\frac{\mathrm{U}_{2 \mathrm{~N}}}{\sqrt{3}}\right)^{2}}{\frac{\mathrm{S}_{\mathrm{N}}}{3 \mathrm{~N}}}\right] \mathrm{X}_{\mathrm{N}} \%}{2 \pi \mathrm{f}}=\frac{\frac{\left(\frac{600}{\sqrt{3}}\right)^{2}}{\left[\frac{3150000}{3 \times 44}\right]} \times 4 \%}{2 \pi \times 50}=0.6 \mathrm{mH}
$$

So the equivalent inductance is:

$$
\mathrm{L}=2 \mathrm{~L}_{1}=1.2 \mathrm{mH}
$$

To ensure that the output voltage ripple is less than $1 \%$, the filter capacitor is selected as follow:

$$
\mathrm{C}=10 \mathrm{mF}
$$

According to condition (1), in order to avoid voltage overshoot the soft-start resistor must satisfy:

$$
\mathrm{R}_{\text {soft }}>K^{2} \sqrt{\frac{L}{C}}=111 \Omega
$$

According to condition (2), in order to limit the maximum charging current the soft-start resistor must satisfy:

$$
5 \Omega=\frac{K^{2} U_{i}}{2 N \times 300}<\mathrm{R}_{\text {soft }}<\frac{300 K}{\sqrt{6} I_{f}} \sqrt{\frac{L}{C}}=1118 \Omega
$$

Actually the soft-start resistor is designed for $300 \Omega$ in the HVPS for ECRH system. Some experiment results are shown in Fig.8 and Fig.9. 


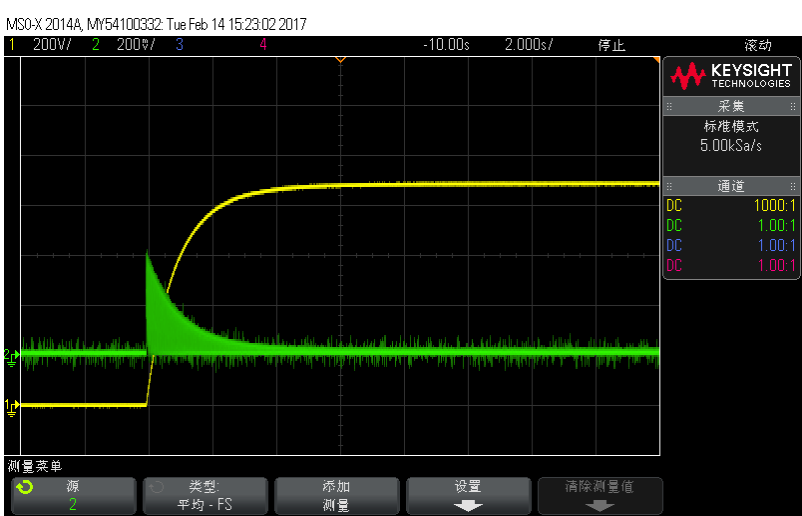

Fig. 8. The capacitor charging waveform with the softstart resistor.

$\mathrm{X}$-axis: $2 \mathrm{~s} / \mathrm{div}$

Y-axis: $200 \mathrm{~V} / \mathrm{div}, 8 \mathrm{~A} / \mathrm{div}$

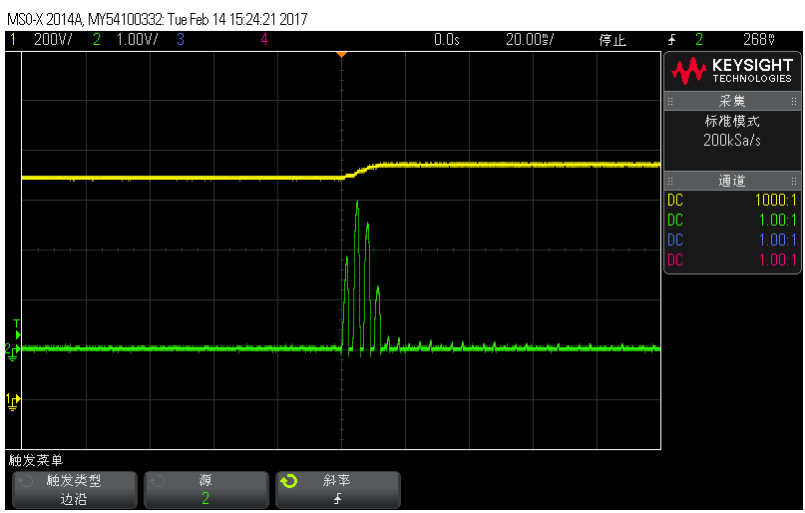

Fig. 9.The capacitor charging waveform of removing the soft-start resistor.

X-axis: 2s/div

Y-axis: $200 \mathrm{~V} / \mathrm{div}, 40 \mathrm{~A} / \mathrm{div}$

In Fig. 8 , the maximum value of charging current with the resistor is $16 \mathrm{~A}$ and the first steady state voltage is $870 \mathrm{~V}$. In Fig.9, the maximum current value of removing the resistor is $110 \mathrm{~A}$ and the second steady state voltage is about $930 \mathrm{~V}$. The voltage change of the capacitor is about $60 \mathrm{~V}$.

According to (7) and (12), the calculation for the maximum value of charging current is $11 \mathrm{~A}$ and $93 \mathrm{~A}$ for different process. According to (11), the voltage change is $64 \mathrm{~V}$. The experimental results are in agreement with the theoretical analysis.

\section{Conclusion}

The soft-start process of HVPS based on PSM technology is studied in this paper. A simplified DC equivalent model is proposed, and the transient and steady state responses of the soft-start process are analysed in detail. The qualifications are obtained and proved by simulation results. The soft-start resistor has been designed for the HVPS of the $140 \mathrm{GHz}$ ECRH system, and the final experimental results show that it is coincident with the theoretical analysis.

This work was supported by the National Mangenetic Confinement Fusion Science Program of China under contract No. 2015GB103000, the National Key Research \& Development Plan 2017YFE0300401, and CAS Key Technology Talent Program.

\section{References}

1. X. Hao, et al., Design and application of a new control system for Tokamak ECRH power supply [J]. Radiation Effects \& Defects in Solids, (3-4):1-10. (2016)

2. J. Zhang, et al., The Design of PSM-Based ECRH Power Supply Control System [J]. Journal of Power \& Energy Engineering, 04(4):91-102. (2016)

3. S. Ma, et al., Analysis and Design of the Module for PSM High-Voltage Power Supply [J]. Journal of Fusion Energy, 34(2):261-266. (2015)

4. S.Ma, et al., High-voltage power supply for ECRH system on J-TEXT Tokamak[C]// Fusion Engineering. IEEE, 1-5. (2013)

5. L. Xia, et al., Analysis of the Soft-Start Circuit of the High Voltage Power Supply Based on PSM Technology [J]. IEEE Transactions on Plasma Science, 42(4):1026-1031. (2014)

6. X. Hao. The Transient Analysis of PSM High Voltage DC Power Supply and the Research of Its Control Strategy [D]. University of Chinese Academy of Sciences. (2012)

7. L.Xia. The Study on Several Key Techniques of High Voltage Power Supply for Auxiliary Heating System in Tokamaks [D]. Huazhong University of Science and Technology (2015) 